\title{
Towards a fully massive five-flavor scheme
}

\author{
Frank Krauss ${ }^{1}$ and Davide Napoletano ${ }^{1,2}$ \\ ${ }^{1}$ Institute for Particle Physics Phenomenology, Durham University, Durham DH1 3LE, United Kingdom \\ ${ }^{2}$ IPhT, CEA Saclay, CNRS UMR 3681, F-91191, Gif-Sur-Yvette, France
}

(Received 10 January 2018; published 5 November 2018)

\begin{abstract}
In this paper, we explore first necessary steps to construct a fully massive version of a variable-flavor number scheme. In particular we focus, as an example, on an extension of the five-flavor scheme, where instead of neglecting explicit initial state quark mass effects, we retain all massive dependence, while keeping the resummation properties of the massless five-flavor scheme. We name this scheme the fiveflavor-massive scheme. Apart from consistently modified parton distribution functions, we provide all the ingredients that are needed to implement this scheme at MC@NLO accuracy, in a Monte Carlo event generator. As proof of concept we implement this scheme in SHERPA and perform a comparison of the new scheme with traditional ones for the simple process of scalar particle production in bottom-quark fusion.
\end{abstract}

DOI: 10.1103/PhysRevD.98.096002

\section{INTRODUCTION}

Processes with heavy quarks (bottom or charm) in the initial state present an interesting challenge for theoretical predictions at the LHC and other hadron collider experiments. First, the finite quark masses introduce another scale to the process, which may or may not prove to be relevant for different observables and different processes. In addition, a decision has to be made in how far heavy quarks can act as incident partons - due to their mass being larger than the QCD scale parameter $m_{Q} \gg \Lambda_{\mathrm{QCD}}$ one could argue that they are disallowed to have a parton distribution function (PDF), thereby decoupling them from the QCD evolution in the initial state, described by the Dokshitzer-GribovLipatov-Altarelli-Parisi (DGLAP) equations. This leads to two complementary solutions. On one hand, heavy quarks $Q$ in the initial and final state may be treated on the same footing as any other light quark, such as the $u, d$, or $s$ quark, by ignoring their mass in the evaluation of the matrix elements. In such a picture the heavy quark acts as an active quark in the QCD evolution equations, and consequently, possibly large collinear logarithms are resummed to all orders into a $Q$ PDF. On the other hand, for some processes and observables, the effects of the finite heavy quark mass $m_{Q}$ become relevant and in such cases these quarks must be treated as fully massive. Traditionally, this immediately translates into the heavy quarks only appearing as final state particles.

Published by the American Physical Society under the terms of the Creative Commons Attribution 4.0 International license. Further distribution of this work must maintain attribution to the author(s) and the published article's title, journal citation, and DOI. Funded by SCOAP ${ }^{3}$.
This dichotomy is most pronounced for the case of $b$-quarks, due to their mass $m_{b} \approx 4.5 \mathrm{GeV}$ being larger than the charm mass by a factor of about 3 . It gives rise to ongoing comparisons of calculations of the same processes and observables in the five- and four-flavor schemes. Here, the former refers to a consistently massless treatment of the $b$-quark, which can therefore be found in both initial and final states, while the latter treats the $b$-quarks as massive and allows them to be in the final state only. For a recent example focusing on the production of $Z$ or Higgs bosons in association with $b$-quarks at the LHC, cf. [1]. There, a slight preference for five-flavor scheme calculations in a multijet merging approach has been found. Broadly speaking, for a wide range of kinematical observables such as the $p_{\perp}$ spectrum of jets or gauge or Higgs bosons away from small momenta, this is in agreement with other similar studies [2-4]. A preferable solution, would be to perform a matching between these two scheme, see for examples [5-14]. However, so far, these schemes have only been worked out for inclusive enough observables and are not yet suitable for a Monte Carlo implementation.

This finding motivates us to extend the five-flavor scheme to allow massive particles in the initial state. In this paper, we present the necessary ingredients for next-toleading-order calculations with massive initial state partons, including these mass effects in an initial state parton shower. We refer to the extended scheme as the five-flavormassive scheme (5FMS). This scheme thus has massive $b$-quarks that contribute both to the running of the coupling constant and to the evolution of PDFs.

There are a number of obstacles to this goal:

(1) In order to calculate cross sections at next-to-leadingorder accuracy in the strong coupling, a scheme to identify, isolate, and subtract infrared divergences is 
yet to be worked out in full detail. In particular, we follow the logic of the Catani-Seymour subtraction formalism, which was first presented for massless partons in [15], later extended to massive fermions in QED in [16], to massive final state QCD partons in [17], and to massive initial state quarks for initial-final dipoles in [18]. We work out phase-space mappings and differential and integrated splitting kernels for the emission of a gluon off a massive quark line in the initial state, with an initial state spectator.

The treatment of massive initial state particles in QED has already been discussed in [16]. However, in contrast to the results there, obtained in $D=4$ dimensions with a massive photon with $m_{\gamma}$ as infrared regulator, we consistently work in $D=4-2 \varepsilon$ dimensions with a massless gluon. Of course, expressions can be mapped onto each other by suitably replacing

$$
e^{2} Q_{a} \sigma_{a} Q_{b} \sigma_{b} \leftrightarrow 4 \pi \alpha_{S} \mu^{2 \varepsilon} \mathbf{T}_{a} \cdot \mathbf{T}_{b},
$$

and, working in the $\overline{\mathrm{MS}}$ scheme,

$$
\log m_{\gamma}^{2} \leftrightarrow \frac{1}{\varepsilon}+\log 4 \pi \mu_{R}^{2}+\mathcal{O}(\varepsilon) .
$$

(2) Standard five-flavor PDFs introduce massive quarks purely perturbatively, through gluon splitting within the evolution. In so doing, special care is devoted to the treatment of threshold effects due to the finite masses, resulting in variable-flavor number schemes, such as the ones detailed in [5-8,10]. However, all these schemes treat mass effects only through thresholds and usually ignore other kinematical effects. We modify standard PDFs through a number of plausible choices detailed below to obtain some handle on the size of such effects. However, a full and comprehensive study of mass effects in PDFs is beyond the scope of this paper. This is also true for more conceptual questions in how far such mass effects must be treated as process-dependent corrections, similar to higher-twist effects. While we acknowledge that these may be important considerations, we leave the detailed study of these effects in hadronhadron collision for a separate work.

An additional problem, as established in [17,19-22], is that starting at next-to-next-to-leading order (NNLO) there are noncanceling infrared divergent contributions that are proportional to the initial state quark mass. This renders the scheme presented in this paper only valid up to next-toleading-order (NLO) accuracy.

Lastly, approaches that use a finite heavy quark mass in the parton shower have been studied in literature [23-25]. Although a comparison is certainly interesting, we leave this to future studies.
The outline of this work is the following. In the next section we very briefly summarize the Catani-Seymour subtraction procedure. There, we also report the ingredients needed to extend this method to include massive initial state quarks. In Sec. III we present the relevant modifications for the matching of the parton shower and next-toleading-order matrix elements. We provide the discussion of results in Sec. IV, where we show explicit results for the production of a scalar boson $A$ in bottom-quark fusion for various combinations of $\left(m_{A}, \tan \beta\right)$ both at fixed order and matched to the parton shower. There we also compare our results with the DIRE parton shower, which includes (collinear) NLO corrections to the DGLAP equation [26,27].

\section{CATANI-SEYMOUR SUBTRACTION FOR MASSIVE INITIAL STATES}

\section{A. Nomenclature}

The differential leading-order (LO) cross section for a hard scattering process with $N$ particles in the final state is given by

$$
\begin{aligned}
\mathrm{d} \hat{\sigma}_{a b}= & \mathrm{d} \Phi_{N}\left(p_{1}, \ldots, p_{N}\right) \mathcal{B}_{N}\left(p_{a}, p_{b} ; p_{1}, \ldots, p_{N}\right) \\
& \times F_{J}^{(N)}\left(p_{1}, \ldots, p_{N} ; p_{a}, p_{b}\right),
\end{aligned}
$$

where $\mathcal{B}$ denotes the Born matrix element squared and the differential phase-space element $\mathrm{d} \Phi_{N}$ implicitly contains the incoming flux of the incident particles, and parton distribution functions, where applicable. Later, where they matter, we will make these factors explicit. The measurement function $F_{J}^{(N)}$ guarantees that the $N$-jet final state is well defined at the Born level and for Born kinematics. In a similar fashion, and suppressing the obvious four-vectors as arguments, the cross section at next-to-leading-order cross section is given by

$\sigma_{N}^{\mathrm{NLO}}=\int \mathrm{d} \Phi_{N}\left[\mathcal{B}_{N}+\mathcal{V}_{N}\right] F_{J}^{(N)}+\int \mathrm{d} \Phi_{N+1} \mathcal{R}_{N} F_{J}^{(N+1)}$,

where the additional terms $\mathcal{V}_{N}$ and $\mathcal{R}_{N}$ signify the virtual and real corrections to the original Born term. They of course relate to final states with $N$ and $N+1$ particles, respectively, as indicated by the phase-space elements. The measurement function must satisfy

$$
\begin{aligned}
F_{J}^{(N+1)}\left(p_{1}, \ldots, k, \ldots, p_{N} ; p_{a}, p_{b}\right) & \rightarrow F_{J}^{(N)}\left(p_{1}, \ldots, p_{N} ; p_{a}, p_{b}\right) \\
\text { if } k \cdot\left\{p_{I}\right\}_{I=i, a, b} & \rightarrow 0 \vee|k| \rightarrow 0
\end{aligned}
$$

to ensure a meaningful cross section definition at NLO accuracy. In later parts of this paper we will assume that this function is implicitly included. 
The soft and collinear divergences related to the emission of the additional particle in $\mathcal{R}_{N}$ are canceled by similar structures in the virtual part $\mathcal{V}_{N}$, but in order to facilitate this cancellation the poles in both must first be isolated and dealt with. Subtraction methods, such as Catani-Seymour subtraction, make use of the universal property of QCD amplitudes in the soft and collinear limits, where the corresponding divergent poles factorize. This allows the construction of process-independent subtraction terms $\mathcal{S}_{N}\left(\Phi_{N} \otimes \Phi_{1}\right)$, such that the first term on the right-hand side of

$$
\begin{aligned}
& \int \mathrm{d} \Phi_{N+1} \mathcal{R}_{N}\left(\Phi_{N+1}\right) \\
& =\int \mathrm{d} \Phi_{N+1}\left[\mathcal{R}_{N}\left(\Phi_{N+1}\right)-\mathcal{S}_{N}\left(\Phi_{N} \otimes \Phi_{1}\right)\right] F_{J}^{(N+1)} \\
& \quad+\int\left[\mathrm{d} \Phi_{N} \otimes \mathrm{d} \Phi_{1}\right] \mathcal{S}\left(\Phi_{N} \otimes \Phi_{1}\right) F_{J}^{(N)}
\end{aligned}
$$

is finite. Assuming that infrared divergences stem from regions in phase space where the momentum $p_{k}$ of a particle $k$ becomes soft or collinear to a particle $i$ with momentum $p_{i}$, the degree of infrared divergence can be parametrized by a small $\lambda \rightarrow 0$ such that $\left|p_{k}\right| \sim \lambda$ or $p_{i} \cdot p_{k} \sim \lambda$. In these divergent phase-space regions, the difference in the first term on the rhs of the equation above behaves as

$\lim _{\lambda \rightarrow 0}\left[\mathcal{R}_{N}\left(\Phi_{N+1}\right)-\mathcal{S}_{N}\left(\Phi_{N} \otimes \Phi_{1}\right)\right]_{p_{k}, p_{i} \cdot p_{k} \sim \lambda}=\mathcal{O}\left(\lambda^{0}\right)$,

i.e., all infrared poles have been canceled.

In addition, the functions $\mathcal{S}\left(\Phi_{N} \otimes \Phi_{1}\right)$ are constructed in such a way that their integral over the extra emission phase space - the second term on the right-hand side of Eq. (2.4) — can be calculated analytically in $D=4+2 \varepsilon$ dimensions, with their divergent parts giving rise to poles $1 / \varepsilon^{2}$ and $1 / \varepsilon$. These poles are ultimately combined with the infrared poles from the loop contributions to cancel exactly.

Combining Eqs. (2.2) and (2.4) yields

$$
\begin{aligned}
\sigma_{N}^{\mathrm{NLO}}= & \int \mathrm{d} \Phi_{N}\left[\mathcal{B}_{N}+\mathcal{V}_{N}+\int \mathrm{d} \Phi_{1} \mathcal{S}_{N}\right] F_{J}^{(N)} \\
& +\int \mathrm{d} \Phi_{N+1}\left[\mathcal{R}_{N}-\mathcal{S}_{N}\right] F_{J}^{(N+1)},
\end{aligned}
$$

where each phase-space integral by itself is infrared finite.

In the Catani-Seymour subtraction, the terms $\mathcal{S}$ are formulated in terms of dipoles, made from three particles, an emitter, a spectator, and the emitted particle $k$. The subtraction term factorizes into a product of processindependent emission terms and Born-like configurations, possibly with parton flavors that differ from the original Born term. These dipoles $\mathcal{D}$ are then classified by the splitter and spectator parton being either in the initial (I) or final (F) state, as II, FI, IF, or FF-the emitted particle $k$ obviously always is in the final state. The overall subtraction term therefore reads

$$
\mathcal{S} \equiv \sum_{i=\mathrm{FF}, \mathrm{FI}, \mathrm{IF}, \mathrm{II}} \mathcal{D}_{i}
$$

where each dipole contribution is given by the sum of all possible emitter-spectator pairs,

$$
\begin{aligned}
& \mathcal{D}_{\mathrm{FF}}=\sum_{(i, j) \neq k} \mathcal{D}_{i j, k}, \quad \mathcal{D}_{\mathrm{FI}}=\sum_{(i, j) \neq k} \mathcal{D}_{i j, k}+\mathcal{D}_{i j}^{a}, \\
& \mathcal{D}_{\mathrm{IF}}=\sum_{i \neq k} \mathcal{D}_{k}^{a i}, \quad \mathcal{D}_{\mathrm{II}}=\mathcal{D}^{a k ; b}+\mathcal{D}^{b k ; a} .
\end{aligned}
$$

In the context of this study we primarily focus on II configurations, which can most conveniently be studied in quark-annihilation processes such as $b \bar{b} \rightarrow H$ and similar. For these processes,

$$
\begin{aligned}
\mathcal{S}= & \mathcal{D}^{a k ; b}\left(p_{1}, \ldots, p_{k}, \ldots, p_{N+1} ; p_{a}, p_{b}\right) \\
& +\mathcal{D}^{b k ; a}\left(p_{1}, \ldots, p_{k}, \ldots, p_{N+1} ; p_{a}, p_{b}\right) .
\end{aligned}
$$

The term $\mathcal{D}^{a k ; b}$

$$
\begin{aligned}
\mathcal{D}^{a k ; b} & \left(p_{1}, \ldots, p_{k}, \ldots, p_{N+1} ; p_{a}, p_{b}\right) \\
= & -\frac{1}{2 x_{a b} p_{a} \cdot p_{k}} \frac{\mathbf{T}_{a} \cdot \mathbf{T}_{b}}{\mathbf{T}_{a}^{2}} \mathbf{V}^{a k, b}\left(p_{a}, p_{b}, p_{k}\right) \\
& \otimes\left|\tilde{\mathcal{M}}_{N}\left(\tilde{p}_{1}, \ldots, \tilde{p}_{N+1} ; \tilde{p}_{a}, p_{b}\right)\right|^{2}
\end{aligned}
$$

represents one individual dipole contribution, where the emitter is the initial state particle $a$ and the spectator is the other initial state particle $b$. The matrix element $\tilde{\mathcal{M}}$ emerges from the original Born-level matrix element by taking into account that the emission of $k$ off parton $a$ might alter the flavor of the resulting parton $\tilde{a}$, and it is evaluated at a kinematical configuration, where the modified fourvectors $\tilde{p}_{a}$ and $\tilde{p}_{i}$ account for four-momentum conservation by absorbing the recoil from emitting $p_{k}$. The functions $\frac{1}{2 p_{i} \cdot p_{j}} \mathbf{V}^{i j, k}\left(p_{i}, p_{k}, p_{j}\right)$ are generically called splitting kernels, or dipole splitting functions, and reduce to the wellknown Altarelli-Parisi [28] splitting kernels $P_{i j}$ in the collinear limit. Note that the $\otimes$ symbol implies possible summation in color and helicity space.

\section{B. Massive II dipoles: Initial state splitter with initial state spectator}

We now present the relevant modification to the described picture, due to the inclusion of finite masses. In the following we make extensive use of the following kinematical quantities: 


$$
\begin{aligned}
s & =\left(p_{a}+p_{b}\right)^{2}, \\
Q^{2} & =\left(p_{a}+p_{b}-p_{k}\right)^{2}=s-2\left(p_{a}+p_{b}\right) \cdot p_{k}, \\
x_{a b} & =\frac{p_{a} \cdot p_{b}-p_{a} \cdot p_{k}-p_{b} \cdot p_{k}}{p_{a} \cdot p_{b}}, \quad y_{a}=\frac{p_{a} \cdot p_{k}}{p_{a} \cdot p_{b}}, \\
s_{a b} & =s-m_{a}^{2}-m_{b}^{2}, \quad \lambda_{a b}=\lambda\left(s, m_{a}^{2}, m_{b}^{2}\right),
\end{aligned}
$$

where

$\lambda(a, b, c)=a^{2}+b^{2}+c^{2}-2(a b+b c)-2 a c$.

The only dipoles involving massive partons which exhibit infrared divergences are those corresponding to the emission of a gluon into the final state. Therefore, we only have to find a suitable expression for the term $\mathbf{V}^{a k, b}\left(p_{a}, p_{b}, p_{k}\right)$ for the case where a heavy initial quark $Q$ emits a gluon with momentum $p_{k}$. In analogy to the treatment for massive final state particles in [17] this is given by

$$
\begin{aligned}
& \mathbf{V}_{a}^{Q_{a} g_{k}, b}\left(p_{a}, p_{b}, p_{k}\right) \\
& \quad=8 \pi \mu^{2 \varepsilon} \alpha_{s} C_{F}\left[\frac{2}{1-x_{a b}}-\left(1+x_{a b}\right)-\varepsilon\left(1-x_{a b}\right)-\frac{x_{a b} m_{a}^{2}}{p_{a} \cdot p_{k}}\right] .
\end{aligned}
$$

In any other case $(g \rightarrow Q \bar{Q}$ and $Q \rightarrow g Q)$ there are no singular contributions rendering the need for subtraction obsolete. It is clear, however, that the collinear divergences present for the very same splittings in the massless case give rise to logarithmically enhanced terms of the form $\log m_{Q}^{2} / \mu^{2}$, where $\mu$ is some large scale related to the dipole kinematics. While one may be tempted to use subtraction terms to smooth these structures and make them more amenable to numerical integration, we have tested explicitly that they do not pose any problem for processes at LHC energies and masses down to $1 \mathrm{GeV}$. In any case, detailed expressions in the QED case in four space-time dimensions can be found in [29].

Coming back to the case of gluon emissions off a heavy quark line, we set the subtraction term to zero for $x_{a b}<\alpha$, the kinematical lower bound,

$$
\alpha=\frac{2 m_{a}^{2} m_{b}^{2}}{s_{a b}} .
$$

In a next step we need to define the phase-space map, connecting the original momenta $\left\{p_{i}\right\}$ of the real emission configuration to the modified momenta $\left\{\tilde{p}_{i}\right\}$ for the reduced matrix element in the subtraction term. This map has to preserve mass-shell conditions, and in particular $\tilde{p}_{a}^{2}=p_{a}^{2}=m_{a}^{2}$, and it is also customary to keep the spectator momentum fixed. As a consequence of these conditions, all other final state momenta $\tilde{p}_{j}$ and their total momentum $\tilde{Q}=\sum_{j} \tilde{p}_{j}$ absorb the recoil in the reduced matrix element.

The transformations are given by

$$
\begin{aligned}
\tilde{p}_{a}^{\mu} & =\sqrt{\frac{\lambda\left(Q^{2}, m_{a}^{2}, m_{b}^{2}\right)}{\lambda_{a b}}}\left(p_{a}^{\mu}-\frac{s_{a b}}{2 m_{b}^{2}} p_{b}^{\mu}\right)+\frac{Q^{2}-m_{a}^{2}-m_{b}^{2}}{2 m_{b}^{2}} p_{b}^{\mu}, \\
\tilde{Q}^{\mu} & =\tilde{p}_{a}^{\mu}+p_{b}^{\mu}, \\
\left.\tilde{p}_{i}^{\mu}\right|_{i \neq k} & =\left.\Lambda_{\nu}^{\mu} p_{i}^{\nu}\right|_{i \neq k},
\end{aligned}
$$

where the Lorentz transformation $\Lambda_{\nu}^{\mu}$ is given by

$$
\Lambda_{\nu}^{\mu}=g_{\nu}^{\mu}-\frac{(Q+\tilde{Q})^{\mu}(Q+\tilde{Q})_{\nu}}{Q^{2}+Q \cdot \tilde{Q}}+\frac{2 \tilde{Q}^{\mu} Q_{\nu}}{Q^{2}}
$$

and applied to all final state particles apart from $k$, including colorless ones.

It is straightforward to check that these relations fulfil the mass-shell conditions, such that $\tilde{p}_{a}^{2}=m_{a}^{2}$ and $\tilde{Q}^{2}=Q^{2}$, and that they possess the right infrared and collinear asymptotic limits.

\section{Phase space}

The phase space for the real emission correction factorizes into a Born-level part and a one-particle phase-space integral,

$$
\begin{aligned}
& \int \mathrm{d} \Phi_{N+1}\left(p_{k}, Q ; p_{a}+p_{b}\right) \\
& =\int_{\alpha}^{1} \mathrm{~d} x \int \mathrm{d} \Phi_{N}\left(\tilde{Q}(x) ; \tilde{p}_{a}(x)+p_{b}\right) \int\left[\mathrm{d}^{d-1} p_{k}\left(s, x, y_{a}\right)\right],
\end{aligned}
$$

where $x$-dependent momenta can be obtained from $\tilde{p}_{a}$ and $\tilde{Q}$ upon replacing $Q^{2} \rightarrow s_{a b} x+m_{a}^{2}+m_{b}^{2}$. The extra particle phase space reads

$$
\begin{aligned}
\int & {\left[\mathrm{d}^{d-1} p_{k}\left(s, x, y_{a}\right)\right] } \\
= & \frac{1}{16 \pi^{2}} \frac{(4 \pi)^{\varepsilon}}{\Gamma(1-\varepsilon)}\left(\frac{s_{a b}}{\sqrt{\lambda_{a b}}}\right)^{1-2 \varepsilon}(1-x)^{1-2 \varepsilon} s_{a b} s^{-\varepsilon} \\
& \times \int_{v_{2}}^{v_{1}} \mathrm{~d} v\left[\left(v_{1}-v\right)\left(v-v_{2}\right)\right]^{-\varepsilon},
\end{aligned}
$$

where, for convenience, we define $v=y_{a} /(1-x)$, and

$$
v_{1,2}=\frac{s_{a b}+2 m_{a}^{2} \mp \sqrt{\lambda_{a b}}}{2 s} .
$$

The integrated splitting function $\widetilde{\mathcal{V}}_{N}^{a, b}$ is given by

$$
\frac{\alpha_{s} C_{F}}{2 \pi} \widetilde{\mathcal{V}}_{N}^{a, b}(x ; \varepsilon)=\int\left[\mathrm{d}^{d-1} p_{k}\left(s, x, y_{a}\right)\right] \frac{1}{2 p_{a} \cdot p_{k}} \mathbf{V}^{a k, b}
$$


and can be decomposed according into an end-point contribution $\mathcal{V}_{N}^{a, b}(\varepsilon)$ containing the $1 / \varepsilon$ pole and a finite part $K^{a, b}(x)$

$$
\widetilde{\mathcal{V}}_{N}^{a, b}(x ; \varepsilon)=\delta(1-x) \mathcal{V}_{N}^{a, b}(\varepsilon)+\left[K^{a, b}(x)\right]_{+} .
$$

The individual pieces read

$$
\begin{aligned}
\mathcal{V}_{N}^{a, b}(\varepsilon) & =\frac{1}{\varepsilon}\left(1+\frac{s_{a b}}{\sqrt{\lambda_{a b}} \log \beta_{0}}\right)+\log \left(\frac{\mu^{2} s}{s_{a b}^{2}}\right)+\frac{3}{2}+\frac{s_{a b}}{\sqrt{\lambda_{a b}}}\left\{\left[\log \left(\frac{\mu^{2} \lambda_{a b}}{m_{a}^{2} s_{a b}^{2}}\right)+\left(\frac{1}{2}-\frac{2 m_{a}^{2}}{s_{a b}}\right)\right] \log \beta_{0}+2 \operatorname{Li}_{2}\left(\beta_{0}\right)+\frac{1}{2} \log ^{2} \beta_{0}-\frac{\pi^{2}}{3}\right\} \\
K^{a, b}(x) & =-\frac{s_{a b}}{\sqrt{\lambda_{a b}}}\left(\frac{1+x^{2}}{1-x}\right) \log \beta_{0}-2 x
\end{aligned}
$$

where

$$
\beta_{0}=\frac{s_{a b}+2 m_{a}^{2}-\sqrt{\lambda_{a b}}}{s_{a b}+2 m_{a}^{2}+\sqrt{\lambda_{a b}}} .
$$

Note that due to the absence of a collinear divergence — which is shielded by the finite quark mass - there are no terms $\propto 1 / \varepsilon^{2}$.

The contribution to the partonic differential cross section is thus given by

$$
\int \mathrm{d} \Phi_{1} \mathcal{S}=\frac{\alpha_{S} C_{F}}{2 \pi}\left\{\left[\mathcal{V}_{N}^{a, b}(\varepsilon)-\int_{0}^{\alpha} \mathrm{d} x K^{a, b}(x)\right] \mathrm{d} \Phi_{N} \mathcal{B}\left(s_{a b}\right)+\int_{\alpha}^{1} \mathrm{~d} x K^{a, b}(x)\left[\frac{\phi\left(x s_{a b}\right)}{x \phi\left(s_{a b}\right)} \mathrm{d} \Phi_{N} \mathcal{B}_{N}\left(x s_{a b}\right)-\mathrm{d} \Phi_{N} \mathcal{B}_{N}\left(s_{a b}\right)\right]\right\},
$$

where we make explicit the dependence on the initial state flux $\phi$.

To embed this into the calculation of cross section at hadron colliders, the PDFs must be added. Parametrizing the incoming hadron and parton momenta as

$$
P_{1,2}^{\mu}=\frac{\sqrt{S}}{2}(1,0,0, \pm 1), \quad p_{a, b}^{\mu}=\eta_{1,2} P_{1,2}+, \frac{m_{a, b}^{2}}{\eta_{1,2} S} P_{2,1}
$$

yields the allowed intervals for the light-cone momentum fractions

$$
\eta_{1,2} \in\left[\frac{1}{2}\left(1-\sqrt{1-\frac{4 m_{a}^{2} m_{b}^{2}}{S^{2}}}\right), \frac{1}{2}\left(1+\sqrt{1-\frac{4 m_{a}^{2} m_{b}^{2}}{S^{2}}}\right)\right] .
$$

Making explicit the flux $\phi\left(s_{a b}\right)=4 \sqrt{\lambda\left(s, m_{a}^{2}, m_{b}^{2}\right)}$, the integral over the incoming light-cone momenta, and the parton distribution functions, the integrated splitting function, corresponding to the purely partonic expression in Eq. (2.24), reads

$$
\begin{aligned}
\mathcal{I}= & \frac{\alpha_{s} C_{F}}{2 \pi} \int \mathrm{d} \eta_{1} \mathrm{~d} \eta_{2} f_{a}\left(\eta_{1}\right) f_{b}\left(\eta_{2}\right)\left\{\left[\mathcal{V}_{N}^{a, b}(\varepsilon)-\int_{0}^{\alpha} \mathrm{d} x K^{a, b}(x)\right] \mathrm{d} \Phi_{N} \mathcal{B}\left(s_{a b}\right)\right. \\
& \left.+\int_{\alpha}^{1} \mathrm{~d} x K^{a, b}(x)\left[\frac{\phi\left(x s_{a b}\right)}{x \phi\left(s_{a b}\right)} \mathrm{d} \Phi_{N} \mathcal{B}_{N}\left(x s_{a b}\right)-\mathrm{d} \Phi_{N} \mathcal{B}_{N}\left(s_{a b}\right)\right]\right\}
\end{aligned}
$$

In this form $\mathcal{I}$ is not very useful for direct implementation, because it implies that the parton-level Born cross section must be integrated over all values of $x$ in the interval $[\alpha, 1]$. To remedy this, we need to rewrite this term, to disentangle the Born cross section from the $x$-integration. To fix this we define the following variable transformation,

$$
x s_{a b}\left(\eta_{1}, \eta_{2}\right)=s_{a b}\left(\eta_{1}^{\prime}(x), \eta_{2}^{\prime}\right) \quad \text { and } \quad \eta_{2}^{\prime}=\eta_{2},
$$

which defines a Jacobean $J\left(\eta_{1}^{\prime}(x), \eta_{2}\right)$, and 


$$
\mathrm{d} \eta_{1} \mathrm{~d} \eta_{2} \mathrm{~d} x=\mathrm{d} \eta_{1}^{\prime} \mathrm{d} \eta_{2} \mathrm{~d} x J\left(\eta_{1}^{\prime}(x), \eta_{2}\right)
$$

where

$$
J\left(\eta_{1}^{\prime}(x), \eta_{2}\right)=\frac{\eta_{1}^{\prime 2}-\frac{m_{a}^{2} m_{b}^{2}}{\eta_{2}^{2} S^{2}}}{2 \eta_{1}^{\prime 2} x}\left\{\frac{\eta_{1}^{\prime 2}+\frac{m_{a}^{2} m_{b}^{2}}{\eta_{2}^{2} S^{2}}+\sqrt{\left(\frac{m_{a}^{2} m_{b}^{2}}{\eta_{2}^{2} S^{2}}+\eta_{1}^{\prime 2}\right)^{2}-4 \frac{m_{a}^{2} m_{b}^{2}}{\eta_{2}^{2} S^{2}} \eta_{1}^{\prime 2} x^{2}}}{\sqrt{\left(\frac{m_{a}^{2} m_{b}^{2}}{\eta_{2}^{2} S^{2}}+\eta_{1}^{\prime 2}\right)^{2}-4 \frac{m_{a}^{2} m_{b}^{2}}{\eta_{2}^{2} S^{2}} \eta_{1}^{\prime 2} x^{2}}}\right\} .
$$

Note that the Jacobean reduces to the usual $1 / x$ factor in the massless limit.

After reversing the integration order and performing the change of variable, we find

$$
\begin{aligned}
& \int \mathrm{d} \eta_{1} \mathrm{~d} \eta_{2} f_{a}\left(\eta_{1}\right) f_{b}\left(\eta_{2}\right) \int_{\alpha}^{1} \mathrm{~d} x K^{a, b}(x) \frac{\phi\left(x s_{a b}\right)}{x \phi\left(s_{a b}\right)} \mathrm{d} \Phi_{N} \mathcal{B}_{N}\left(x s_{a b}\right) \\
& =\int \mathrm{d} \eta_{1}^{\prime} \mathrm{d} \eta_{2} f_{a}\left(\eta_{1}^{\prime}\right) f_{b}\left(\eta_{2}\right) \mathrm{d} \Phi_{N} \mathcal{B}_{N}\left(s_{a b}\right) \int_{\bar{\alpha}}^{1} \mathrm{~d} x K^{a, b}(x)\left[J\left(\eta_{1}, \eta_{2}\right) \frac{\phi\left(s_{a b}\right)}{x \phi\left(s_{a b}\left(\eta_{1}\right)\right)} \frac{f_{a}\left(\eta_{1}\right)}{f_{a}\left(\eta_{1}^{\prime}\right)}\right],
\end{aligned}
$$

where $\eta_{1}=\eta_{1}\left(\eta_{1}^{\prime}, x\right)$ is the old variable expressed in terms of the new ones, and where the integration boundary is now given by

$$
\bar{\alpha}=\max \left\{\alpha, \eta_{1}^{\prime}\right\} .
$$

Renaming $\eta_{1} \leftrightarrow \eta_{1}^{\prime}$ finally yields

$\mathcal{I}=\frac{\alpha_{s} C_{F}}{2 \pi} \int \mathrm{d} \eta_{1} \mathrm{~d} \eta_{2} f_{a}\left(\eta_{1}\right) f_{b}\left(\eta_{2}\right) \mathrm{d} \Phi_{N} \mathcal{B}_{N}\left(s_{a b}\right)\left\{\mathcal{V}_{N}^{a, b}(\varepsilon)+\int_{\bar{\alpha}}^{1} \mathrm{~d} x K^{a, b}(x)\left[J\left(\eta_{1}^{\prime}, \eta_{2}\right) \frac{\phi\left(s_{a b}\right)}{x \phi\left(s_{a b}\left(\eta_{1}^{\prime}\right)\right)} \frac{f_{a}\left(\eta_{1}^{\prime}\right)}{f_{a}\left(\eta_{1}\right)}-1\right]-\int_{0}^{\bar{\alpha}} \mathrm{d} x K^{a, b}(x)\right\}$.

This disentangles the evaluation of the Born cross section from the $x$-integral such that the whole curly bracket in Eq. (2.33) acts as a local $K$-factor on top of the partonic cross section.

\section{Dipole formulas for initial-final configurations}

A detailed derivation of dipole formulas in the initial-final and final-initial cases can be found in [18], although in a slightly different notation compared to the one presented in this work. In principle one could also extract all relevant formulas from [16] with the modifications described in the Introduction, following the steps presented in the previous section.

We consider the splitting $Q_{a} \rightarrow g_{k} Q$ with spectator $i$ in the final state. To make the reading of this section more transparent, we also report some useful kinematical quantities used throughout

$$
\begin{aligned}
Q^{2} & =\left(p_{i}-p_{a}+p_{k}\right)^{2}, \quad x_{a i}=\frac{p_{a} \cdot p_{i}+p_{a} \cdot p_{k}-p_{i} \cdot p_{k}}{p_{a} \cdot p_{i}+p_{a} \cdot p_{k}}, \quad y_{a}=\frac{p_{a} \cdot p_{i}}{p_{a} \cdot p_{i}+p_{a} \cdot p_{k}}, \\
R_{a i}(x) & =\sqrt{\frac{\left(Q^{2}+2 m_{a}^{2} x\right)^{2}-4 m_{a}^{2} Q^{2} x^{2}}{\lambda_{a i}}}, \quad \lambda_{a i}=\lambda\left(Q^{2}, m_{a}^{2}, m_{i}^{2}\right) .
\end{aligned}
$$

The subtraction term in this case is given by

$\mathcal{D}_{k}^{a i}\left(p_{1}, \ldots, p_{i}, \ldots, p_{k}, \ldots, p_{N+1} ; p_{a}, p_{b}\right)=-\frac{1}{2 x_{a i} p_{a} \cdot p_{k}} \frac{\mathbf{T}_{a} \cdot \mathbf{T}_{i}}{\mathbf{T}_{a}^{2}} \mathbf{V}_{i}^{a k}\left(p_{a}, p_{i}, p_{k}\right) \otimes\left|\tilde{\mathcal{M}}_{N}\left(\tilde{p}_{1}, \ldots, \tilde{p}_{N+1} ; \tilde{p}_{a}, p_{b}\right)\right|^{2}$,

where the only divergent dipole contribution in the massive case reads 


$$
\mathbf{V}_{i}^{Q_{a} g_{k}}\left(p_{a}, p_{i}, p_{k}\right)=8 \pi \mu^{2 \varepsilon} \alpha_{s} C_{F}\left[\frac{2}{2-x_{a i}-z_{a i}}-R_{a i}\left(x_{a i}\right)\left(1+x_{a i}\right)-\varepsilon\left(1-x_{a b}\right)-\frac{x_{a i} m_{a}^{2}}{p_{a} \cdot p_{k}}\right]
$$

The mapped momenta can be expressed in terms of the original momenta using

$$
\tilde{p}_{i}^{\mu}=\sqrt{\frac{\lambda_{a i}}{\lambda\left(\left(p_{i}+k\right)^{2}, Q^{2}, m_{a}^{2}\right)}}\left(p_{a}^{\mu}-\frac{Q \cdot p_{a}}{Q^{2}} Q^{\mu}\right)+\frac{Q^{2}-m_{a}^{2}+m_{i}^{2}}{2 Q^{2}} Q^{\mu}, \quad \tilde{p}_{a}^{\mu}=\tilde{p}_{i}^{\mu}-Q^{\mu} .
$$

The integral of the extra emission phase space can be split into two contributions, as done in Eq. $(2.21)$, $\mathcal{V}_{i, N}^{a}(\varepsilon)$ and $\left[K_{i}^{a}(x)\right]_{+}$. They are given by

$$
\begin{aligned}
\mathcal{V}_{i, N}^{a}(\varepsilon)= & \frac{1}{\varepsilon}\left(1+\frac{Q^{2}}{\sqrt{\lambda_{a i}}} \log \frac{c_{1}}{c_{0}}\right)+\log \left(\frac{\mu^{2} m_{i}^{2}}{Q^{4}}\right)+\frac{1}{2}+\frac{Q^{2}}{\sqrt{\lambda_{a i}}}\left[\log \left(\frac{c_{1}}{c_{0}}\right) \log \left(\frac{m_{a}^{2} \mu^{2}}{b_{0}^{2} \lambda_{a i}}\right)-\log \left(c_{1}\right) \log \left(\frac{m_{a}^{2}+m_{i}^{2}-Q^{2}}{m_{a}^{2}}\right)\right. \\
& \left.+\frac{1}{2} \log \left(\frac{c_{1}}{c_{0}}\right) \log \left(c_{0} c_{1}\right)-\frac{\log \left(c_{0}\right)}{2}-2 \sum_{k=0}^{5}(-1)^{k} \operatorname{Li}_{2}\left(c_{k}\right)\right]+\frac{Q^{4}\left(2 m_{i}^{2}+3 Q^{2}\right)}{2 \sqrt{\lambda_{a i}}\left(m_{i}^{2}+Q^{2}\right)^{2}}\left[\log \left(\frac{\sqrt{\lambda_{a i}}-2 m_{a}^{2}-2 \gamma^{2} Q^{2}+Q^{2}}{2 \gamma^{2} m_{i}^{2}}\right)\right. \\
& \left.+\frac{1}{\gamma} \log \left(\frac{\gamma\left(\sqrt{\lambda_{a i}}+\gamma Q^{2}\right)+2 m_{a}^{2}}{(\gamma-1) \gamma Q^{2}}\right)+\frac{\left(\sqrt{\lambda_{a i}}+Q^{2}\right)\left(m_{i}^{2}+Q^{2}\right)}{2 m_{a}^{2}\left(2 m_{i}^{2}+3 Q^{2}\right)}\right],
\end{aligned}
$$

where

$$
\begin{aligned}
c_{0} & =\frac{Q^{2}+\sqrt{\lambda_{a i}}}{Q^{2}-\sqrt{\lambda_{a i}}}, \quad c_{1}=\frac{Q^{2}-2 m_{i}^{2}-\sqrt{\lambda_{a i}}}{Q^{2}-2 m_{i}^{2}-\sqrt{\lambda_{a i}}} \\
c_{2,3} & =\frac{ \pm \sqrt{\lambda_{a i}}-Q^{2}-2 m_{a}^{2}}{2 m_{a}^{2}}, \quad c_{4,5}=2 b_{0} \frac{m_{i}^{2}-m_{a}^{2} \mp \sqrt{\lambda_{a i}}}{2 m_{a}^{2}-Q^{2} \mp \sqrt{\lambda_{a i}}} \\
b_{0} & =\frac{2 m_{a}^{2}}{\sqrt{\lambda_{a i}}-Q^{2}-2 m_{a}^{2}}, \quad \gamma=\frac{m_{a}^{2}}{\sqrt{-Q^{2}-m_{i}^{2}+i \epsilon}},
\end{aligned}
$$

and

$$
\begin{aligned}
K_{i}^{a}(x)= & -\frac{Q^{2}}{\sqrt{\lambda_{a i}}} \frac{1}{R_{a i}(x)}\left\{\frac{2}{1-x} \log \frac{\left[1-z_{1}(x)\right]\left[2-x-z_{2}(x)\right]}{\left[1-z_{2}(x)\right]\left[2-x-z_{1}(x)\right]}+R_{a i}(x)(1+x) \log \frac{1-z_{2}(x)}{1-z_{1}(x)}\right. \\
& \left.+\frac{2 m_{a}^{2} x^{2}}{Q^{2}}\left[\frac{1}{1-z_{2}(x)}-\frac{1}{1-z_{1}(x)}\right]\right\}
\end{aligned}
$$

with

$$
z_{1,2}(x)=\frac{\left[Q^{2}-x\left(Q^{2}+2 m_{i}^{2}\right)\right] \mp R_{a i}(x)(1-x)^{2} \sqrt{\lambda_{a i}}}{2\left[Q^{2}-x\left(Q^{2}-m_{a}^{2}\right)\right]}
$$

The rest of the derivation follows exactly as in the previous section.

\section{MC@NLO MATCHING}

Having successfully built fixed-order NLO matrix elements in the 5FMS, we now proceed to the matching to the parton shower along the lines of the well-established MC@NLO technique [30] as implemented with small variations in the SHERPA Monte Carlo [31], and referred to as S-MC@NLO [32-34].
Note that our implementation closely follows that of [32,33], which we refer to for further details. We start by constructing the NLO-weighted Born cross section,

$$
\begin{aligned}
\overline{\mathcal{B}}\left(\Phi_{N}\right)= & \mathcal{B}\left(\Phi_{N}\right)+\mathcal{V}\left(\Phi_{N}\right)+\mathcal{I}\left(\Phi_{N}\right) \\
& +\int \mathrm{d} \Phi_{1}\left[D^{(A)}\left(\Phi_{N+1}\right) \Theta\left(\mu_{Q}^{2}-t\left(\Phi_{N+1}\right)\right)\right. \\
& \left.-D^{(S)}\left(\Phi_{N+1}\right)\right]
\end{aligned}
$$

where we have defined $D^{(A)}\left(\Phi_{N+1}\right)$ such that $\mathcal{R}_{N}\left(\Phi_{N+1}\right)$ can be split into an unresolved divergent part and a hard resolved part, 


$$
\mathcal{R}_{N}\left(\Phi_{N+1}\right)=D^{(A)}\left(\Phi_{N+1}\right)+\mathcal{H}\left(\Phi_{N+1}\right)
$$

and redefined Eq. (2.7) $\mathcal{S} \equiv D^{(S)}$ to be consistent with the notation commonly used in this context. It is worth mentioning at this stage that $D^{(A),(S)}$ have the same formal structure and they only differ by finite terms. ${ }^{1}$ As a consequence they can both be written using the structure of Eqs. (2.7) and (2.8),

$$
D^{(A),(S)} \equiv \sum_{i=\mathrm{FF}, \mathrm{FI}, \mathrm{IF}, \mathrm{II}} \mathcal{D}_{i}^{(A),(S)}
$$

The last ingredient needed is the MC@NLO Sudakov form factor. This is built starting from $D^{(A)}\left(\Phi_{N+1}\right)$,

$$
\Delta^{(A)}\left(t, t^{\prime}\right)=\exp \left\{-\int_{t}^{t^{\prime}} \mathrm{d} \phi_{1} \frac{D^{(A)}\left(\Phi_{N+1}\right)}{\mathcal{B}\left(\Phi_{N}\right)}\right\}
$$

In particular, Eq. (2.7) implies that the Sudakov form factor can be decomposed as

$$
\begin{aligned}
\Delta^{(A)}\left(t, t^{\prime}\right) & =\exp \left\{-\sum_{i=\mathrm{FF}, \mathrm{FI}, \mathrm{IF}, \mathrm{II}} \int_{t}^{t^{\prime}} \mathrm{d} \phi_{1} \frac{D_{i}^{(A)}\left(\Phi_{N+1}\right)}{\mathcal{B}\left(\Phi_{N}\right)}\right\} \\
& \equiv \prod_{i=\mathrm{FF}, \mathrm{FI}, \mathrm{IF}, \mathrm{II}} \Delta_{i}\left(t, t^{\prime}\right) .
\end{aligned}
$$

The inclusion of mass effects in the initial state only modifies the $i=\mathrm{II}$, IF contributions to $\Delta^{(A)}$ with respect to their original definitions, which is what we focus on in the rest of this section.

Finally, the MC@NLO-matched fully differential cross section can be written in terms of the previous ingredients as

$$
\begin{aligned}
\mathrm{d} \sigma^{\mathrm{MC} @ \mathrm{NLO}}= & \mathrm{d} \Phi_{N} \overline{\mathcal{B}}\left(\Phi_{N}\right)\left[\Delta^{(A)}\left(t_{0}, \mu_{Q}^{2}\right)\right. \\
& \left.+\int_{t \in\left[t_{0}, \mu_{Q}^{2}\right]} \mathrm{d} \Phi_{1} \frac{D^{(A)}\left(\Phi_{N+1}\right)}{\mathcal{B}\left(\Phi_{N}\right)} \Delta^{(A)}\left(t\left(\Phi_{1}\right), \mu_{Q}^{2}\right)\right] F_{J}^{(N)} \\
& +\mathrm{d} \Phi_{N+1} \mathcal{H}\left(\Phi_{N+1}\right) F_{J}^{(N+1)}
\end{aligned}
$$

\section{A. Sudakov form factor}

We now describe explicitly how the $\Delta_{i}$ contributions are constructed in our implementation. As already noted, only the $i=\mathrm{II}$, IF are changed with respect to their original implementation, so in the following we restrict our discussion to them. Most of the ingredients relevant to the matching can be obtained as the four-dimensional limit $(\varepsilon \rightarrow 0)$ of the equations presented in Sec. II.

One comment is in order here. The initial state evolution is partially driven by ratios of PDF factors at different scales and possibly for different flavors for transitions of quarks to gluons. This may lead to a situation where such a factor reads $f_{g} / f_{Q}$, i.e., the ratio of a gluon and a heavy quark PDF. Ignoring effects of intrinsic charm and beauty, the quark PDF has no support below its mass threshold, and this ratio becomes ill defined. Different solutions have been constructed in various parton shower algorithms, most of which effectively enforce a splitting such that the heavy quark is replaced by a gluon at threshold.

\section{Initial-initial configurations}

First, we need to express the transverse momentum of the emission-the ordering variable in SHERPA's parton showering - in terms of the variables used to construct the subtraction $x$ and $y$,

$\mathbf{k}_{\perp}^{2}=\frac{2 y(1-x-y) p_{a} \cdot p_{b}-(1-x-y)^{2} m_{a}^{2}-y^{2} m_{b}^{2}}{1-\frac{m_{a}^{2} m_{b}^{2}}{\left(p_{a} \cdot p_{b}\right)^{2}}}$.

Further, we need the relevant Jacobian factor for the oneparticle phase-space integration in Eq. (2.18),

$$
\frac{\mathrm{d} \mathbf{k}_{\perp}^{2}}{\mathbf{k}_{\perp}^{2}}=\frac{1-x-2 y+(1-x-y) \frac{m_{a}^{2}}{p_{a} \cdot p_{b}}-y \frac{m_{b}^{2}}{p_{a} \cdot p_{b}}}{1-x-y-\frac{(1-x-y)^{2}}{2 y} \frac{m_{a}^{2}}{p_{a} \cdot p_{b}}-\frac{y}{2} \frac{m_{b}^{2}}{p_{a} \cdot p_{b}}} .
$$

The fully massive Sudakov form factor for initial-initial dipole configurations is thus given by

$$
\Delta_{\text {II }}\left(\mathbf{k}_{\perp, \max }^{2}, \mathbf{k}_{\perp, 0}^{2}\right)=\exp \left\{-\sum_{a k} \sum_{b \neq a k} \frac{1}{\mathcal{N}_{\text {spec }}} \int_{\mathbf{k}_{\perp, 0}^{2}}^{\mathbf{k}_{\perp, \max }} \frac{\mathrm{d} \mathbf{k}_{\perp}^{2}}{\mathbf{k}_{\perp}^{2}} \int_{x_{-}}^{x_{+}} \mathrm{d} x \mathcal{J}_{\mathrm{II}}\left(x, y ; \mathbf{k}_{\perp}^{2}\right) \mathbf{V}^{a k, b}\left(p_{a}, p_{b}, p_{k}\right)\right\},
$$

where

$$
\mathcal{J}_{\mathrm{II}}\left(x, y ; \mathbf{k}_{\perp}^{2}\right)=\frac{1-x-y-\frac{(1-x-y)^{2}}{2 y} \frac{m_{a}^{2}}{p_{a} \cdot p_{b}}-\frac{y}{2} \frac{m_{b}^{2}}{p_{a} \cdot p_{b}}}{1-x-2 y+(1-x-y) \frac{m_{a}^{2}}{p_{a} \cdot p_{b}}-y \frac{m_{b}^{2}}{p_{a} \cdot p_{b}}} \frac{s_{a b}}{\sqrt{\lambda_{a b}}} \frac{1}{x} \frac{f_{a}(\eta / x)}{f_{a}(\eta)},
$$

and $\mathbf{V}^{a k, b}$ can be taken from Eq. (2.13).

\footnotetext{
${ }^{1}$ In their implementation in SHERPA, in particular, they are equal up to phase space, so that $D^{(A)}\left(\Phi_{N+1}\right)=D^{(S)}\left(\Phi_{N+1}\right) \Theta\left(\mu_{Q}^{2}-\mathbf{k}_{\perp}^{2}\right)$.
} 


\section{Initial-final configurations}

Similar to the previous case, we get

$$
\mathbf{k}_{\perp}^{2}=\frac{2 p_{a} \cdot p_{i}(1-y)(1-x y)-m_{i}^{2}(1-y)^{2}-m_{a}^{2}(1-x y)^{2}}{y^{2}\left(1-\frac{m_{a}^{2} m_{a}^{2}}{\left(p_{a} \cdot p_{i}\right)^{2}}\right)} .
$$

This implies that the Jacobian factor becomes

$$
\mathcal{J}_{\mathrm{IF}}\left(x, y ; \mathbf{k}_{\perp}^{2}\right)=\frac{2(1-y)(1-x y)-(1-y)^{2} \frac{m_{i}^{2}}{p_{a} \cdot p_{i}}-(1-x y)^{2} \frac{m_{a}^{2}}{p_{a} \cdot p_{i}}}{2-y-x y-(1-y) \frac{m_{i}^{2}}{p_{a} \cdot p_{i}}-(1-x y) \frac{m_{a}^{2}}{p_{a} \cdot p_{i}}} \frac{Q^{2}}{\sqrt{\lambda_{a i}}} \frac{1}{x} \frac{f_{a}(\eta / x)}{f_{a}(\eta)},
$$

which in turn gives the fully massive initial-final contribution to the Sudakov form factor

$$
\Delta_{\mathrm{IF}}\left(\mathbf{k}_{\perp, \max }^{2}, \mathbf{k}_{\perp, 0}^{2}\right)=\exp \left\{-\sum_{a k} \sum_{b \neq a k} \frac{1}{\mathcal{N}_{\mathrm{spec}}} \int_{\mathbf{k}_{\perp, 0}^{2}}^{\mathbf{k}_{\perp, \max }} \frac{\mathrm{d} \mathbf{k}_{\perp}^{2}}{\mathbf{k}_{\perp}^{2}} \int_{x_{-}}^{x_{+}} \mathrm{d} x \mathcal{J}_{\mathrm{IF}}\left(x, y ; \mathbf{k}_{\perp}^{2}\right) \mathbf{V}_{i}^{a k}\left(p_{a}, p_{i}, p_{k}\right)\right\},
$$

where $\mathbf{V}_{i}^{a k}\left(p_{a}, p_{i}, p_{k}\right)$ is defined in Eq. (2.36).

\section{B. Physical kinematics}

In the practical implementation of the parton shower procedure, the extra emission is attached to an underlying $N$-particle phase space. This corresponds to the reversed procedure used to construct the reduced matrix elements in Sec. II. The new momenta are then obtained from the old ones by inverting Eqs. (2.37).

\section{Initial-initial configurations}

For initial-initial configurations, we get

$$
\begin{aligned}
p_{a}^{\mu}= & \frac{1}{x}\left[\sqrt{\frac{\left(2 \tilde{p}_{a} \cdot \tilde{p}_{b}\right)^{2}-4 m_{a}^{2} m_{b}^{2} x^{2}}{\lambda\left(\left(\tilde{p}_{a}+\tilde{p}_{b}\right)^{2}, m_{a}^{2}, m_{b}^{2}\right)}}\left(\tilde{p}_{a}^{\mu}-\frac{\tilde{p}_{a} \cdot \tilde{p}_{b}}{m_{b}^{2}} \tilde{p}_{b}^{\mu}\right)\right. \\
& \left.+\frac{\tilde{p}_{a} \cdot \tilde{p}_{b}}{m_{b}^{2}} \tilde{p}_{b}^{\mu}\right], \\
p_{b}^{\mu}= & \tilde{p}_{b}^{\mu}, \\
p_{k}^{\mu}= & \frac{1-x-y-y \frac{m_{b}^{2}}{p_{a} \cdot p_{b}}}{1-\frac{m_{a}^{2} m_{b}^{2}}{\left(p_{a} \cdot p_{b}\right)^{2}}} p_{a}^{\mu}+\frac{y-(1-x-y) \frac{m_{a}^{2} x}{p_{a} \cdot p_{b}}}{1-\frac{m_{a}^{2} m_{b}^{2}}{\left(p_{a} \cdot p_{b}\right)^{2}}} p_{b}^{\mu}+\mathbf{k}_{\perp}^{\mu} .
\end{aligned}
$$

\section{Initial-final configurations}

Similarly, for initial-final configurations,

$$
\begin{aligned}
p_{k}^{\mu}= & \frac{1}{y} \frac{1-x y-(1-y) \frac{m_{i}^{2}}{p_{a} \cdot p_{i}}}{1-\frac{m_{a}^{2} m_{i}^{2}}{\left(p_{a} \cdot p_{i}\right)^{2}}} p_{a}^{\mu} \\
& +\frac{1}{y} \frac{1-y-(1-x y) \frac{m_{a}^{2} x}{p_{a} \cdot p_{i}}}{1-\frac{m_{a}^{2} m_{b}^{2}}{\left(p_{a} \cdot p_{i}\right)^{2}}} p_{i}^{\mu}+\mathbf{k}_{\perp}^{\mu} .
\end{aligned}
$$

All other configurations are left unchanged by our scheme.

\section{RESULTS}

To study the impact of the inclusion of finite-mass effects, we compare results of the 5FMS with the vanilla five-flavor scheme, where $b$-quarks are massless. In particular, just as an example and with no intentions of making any statement about any beyond the Standard Model, we focus on the production of a scalar particle $A$ coupling to $b$ quarks through a Yukawa coupling. As a proxy to test the impact of the inclusion of mass effects we vary the mass of this scalar particle in the range $20-500 \mathrm{GeV}$. Further, we let the coupling of the $b$-quarks to this particle vary too by varying the parameter $\tan \beta$, mimicking a two Higgs doublet model.

We study this process both at fixed-order next-toleading-order accuracy and at MC@NLO accuracy. Plots for the former are collected in Fig. 1, while the latter setup is shown in Fig. 2. All our results are obtained within the SHERPA event generator [31]. Leading-order matrix elements, including those of real radiation processes, are calculated using the AMEGIC++ [35] matrixelement generator. The differential subtraction follows closely the algorithms of [36], extended with the ingredients reported in Sec. II. The integrated subtraction terms are implemented in SHERPA and will be made publicly available in a future SHERPA release. Virtual corrections have been obtained from the OPENLOOPS generator [37]. Finally, for the MC@NLO results, we make use of the modifications described in Sec. III to SHERPA standard parton shower, the CSS shower [38].

We apply no cuts at generation level. However, in the following we define as $b$-jet any jet with $p_{\perp} \geq 25 \mathrm{GeV}$ that has at least one $b$-flavored parton in it. We further require any particle in the final state to have $|\eta| \leq 2.5$. Jets are clustered using the anti- $k_{T}$ [39] algorithm as implemented in FASTJET [40] while the event analysis is performed using the RIVET package [41,42]. We set the 

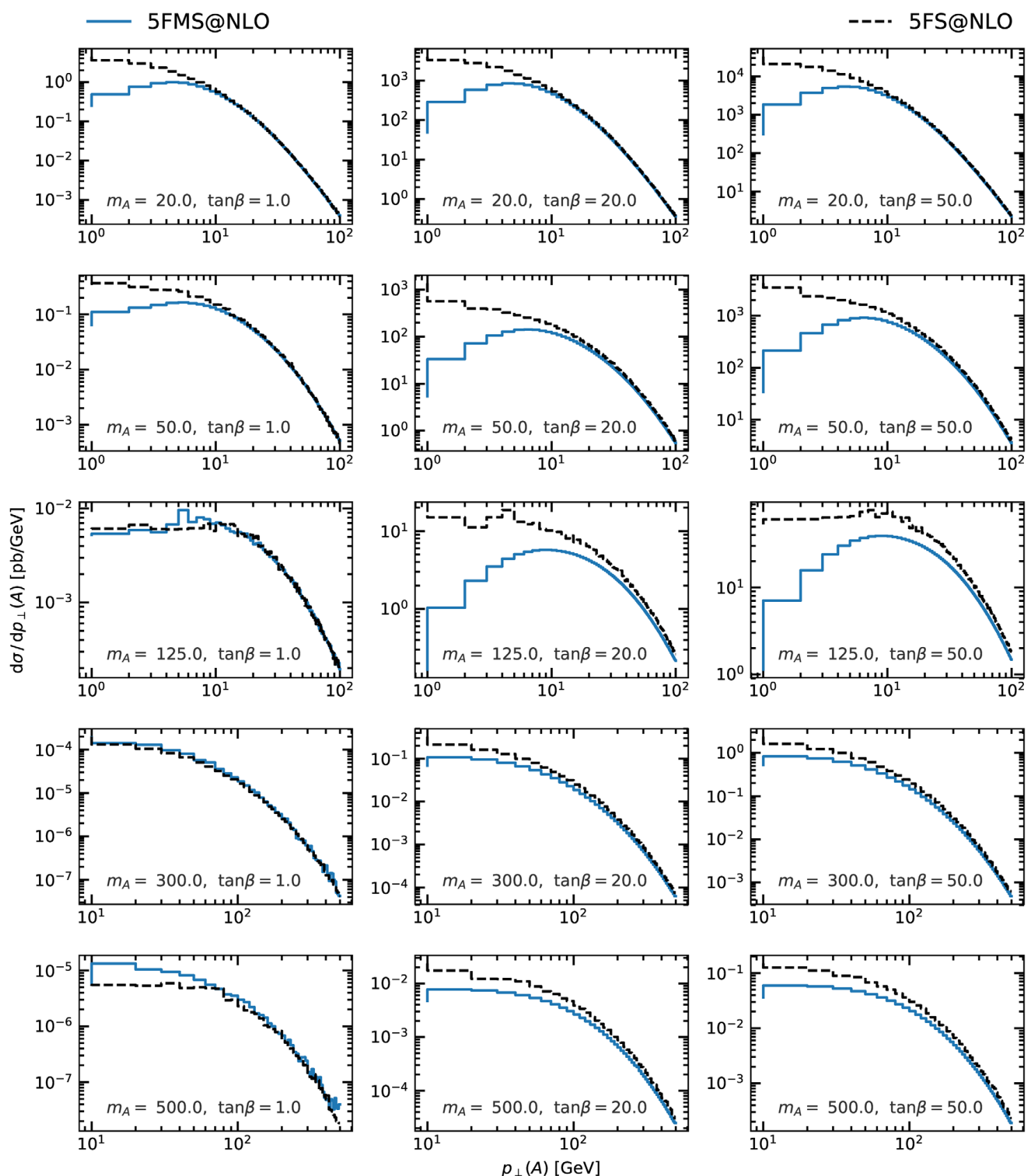

FIG. 1. The $p_{\perp}$ spectrum of the scalar boson $A$ for various combinations of $m_{A}$ and $\tan \beta$, in the 5FS and 5FMS fixed-order predictions.

renormalization, factorization, and shower starting scales to be $\mu_{R}=\mu_{F}=\mu_{Q}=m_{A} / 3$.

For fixed-order predictions, in Fig. 1, we use theSHERPA default-NNPDF30 NNLO set with $\alpha_{s}\left(m_{Z}\right)=$ 0.118 [43] for the 5FS and the 5FMS. For results presented in Fig. 2, however, where we further compare the two fiveflavor scheme predictions with a LO matrix element matched with the NLL DIRE parton shower [26,27], we employ the CT14 NNLO PDF set [44], to satisfy DIRE's requirement of positive definite PDFs, for all predictions. Note that the DIRE prediction is also with five massless flavor, like the standard 5FS.
We start our discussion from the fixed-order results (Fig. 1). In this case, we have two mass scales, $p_{\perp}$ and $m_{A}$. Further, as we let them vary, the mass corrections differ in size in different regions of phase space. In any case, we always expect the 5FS and the 5FMS to converge to each other in the region $p_{\perp} \sim m_{A}$, which is indeed evident in Fig. 1. Note the different $p_{\perp}$ range in Fig. 1 for $m_{A} \geq 300 \mathrm{GeV}$ to show this expected behavior. As $m_{A}$ increases we expect a reduction in the absolute size of mass effects, with differences between the two schemes remaining in the region $p_{\perp} \sim m_{b}$.

Looking at the standard $\left(m_{H}=125, \tan \beta=1\right)$ point, we expect mass effects to play a marginal role, of order 

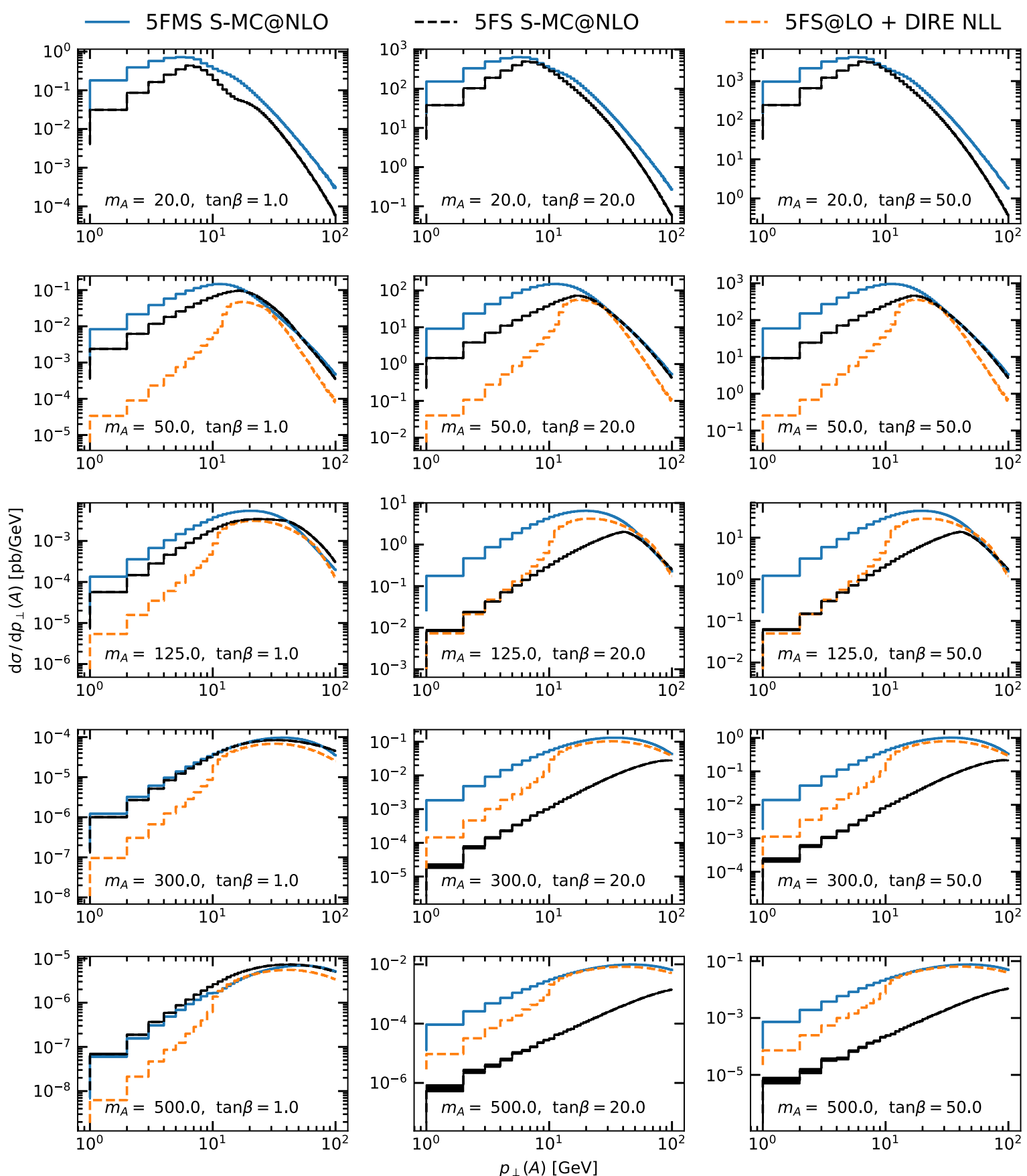

FIG. 2. The $p_{\perp}$ spectrum of the scalar boson $A$ for various combinations of $m_{A}$ and $\tan \beta$. The 5FS and 5FMS are computed at MC@NLO accuracy while the DIRE prediction is obtained matching a LO matrix element with the next-to-leading-log (NLL) shower [26,27].

$\sim 1 \%-5 \%$, at the level of total cross sections. Furthermore, since they are power suppressed, we expect them to be less important at large $p_{\perp}$, while having the largest impact in the lower bins of the distribution. This is due to the fact that the difference in the mass treatment between the two schemes is only in the hard matrix elements and phase space. This is confirmed in our plot.

We now turn to the parton shower-matched results (Fig. 2). Leaving for a second the discussion of the DIRE prediction aside, in general, we expect a shower prediction to fall on the fixed-order result for values of $p_{\perp} \gtrsim \mu_{Q}$, where the discussion of fixed-order results apply. In this case, too, we expect less important mass effects in the region where both $m_{A}$ and $p_{\perp}$ are large, but not for large $m_{A}$ and small $p_{\perp}$. These general considerations are all confirmed in Fig. 2. The additional sample is obtained using DIRE. As the implementation of this shower stops the emission for scales $\sim 2 m_{b}$, no transverse momentum is 
generated for the scalar particle when $m_{A}=20 \mathrm{GeV}$, with $\mu_{Q}=m_{A} / 3<2 m_{b}$. As for all other configurations, the 5FMS and DIRE predictions are in good agreement, in the region of the Sudakov peak $p_{\perp} \lesssim \mu_{Q}$.

\section{CONCLUSIONS}

In this paper we presented all the ingredients that are necessary to construct an extension of Variable-FlavorNumber-Scheme, like the 5FS, in which heavy quarks are treated as massless partons, to allow for massive partons in the initial state, for processes at hadron colliders, at NLO accuracy. In particular we extended the successful CataniSeymour scheme for subtraction of infrared divergences to the case of massive initial states. In variance to an earlier treatment by Dittmaier, we do not use a finite photon mass as regulator but consistently work in $D=4+2 \varepsilon$ dimensions. We also reparametrize the result for the integrated subtraction term in such a way that the residual integral over the light-cone fraction of the emitted particle decouples from the evaluation of the Born cross section, rendering our result more useful for direct implementation. Further, we used these massive dipoles to extend the SMC@NLO matching as well as the shower generation.
We investigated the effect of finite quark masses at fixedorder accuracy in the process $b \bar{b} \rightarrow A$. Mass effects for this process are generally quite small, both at the inclusive and differential level, on the order of a few percent. This however might not hold true for other processes involving heavy quarks. A five-flavor-massive scheme will provide insight by producing fully differential results including mass effects in a consistent way at matrix-element level. As an additional example we presented simulations for the production of a scalar particle $A$ in bottom-quark fusion, for various configurations of $m_{A}$ and $\tan \beta$.

\section{ACKNOWLEDGMENTS}

We want to thank our colleagues from the SHERPA collaboration for animated discussions, technical and moral support. We are particularly grateful to Stefan Höche for his help in the implementation of the method and the running of the DIRE parton shower. This work has received funding from the European Union's Horizon 2020 research and innovation programme as part of the Marie SkodowskaCurie Innovative Training Networks "MCnetITN3" (Grant No. 722104) and "HiggsTools" (Grant No. PITN-GA2012316704) and by the ERC Advanced Grant MC@NNLO (340983).
[1] F. Krauss, D. Napoletano, and S. Schumann, Simulating $b$-associated production of $Z$ and Higgs bosons with the SHERPA event generator, Phys. Rev. D 95, 036012 (2017).

[2] S. Chatrchyan et al. (CMS Collaboration), Measurement of the cross section and angular correlations for associated production of a $\mathrm{Z}$ boson with $b$ hadrons in $p p$ collisions at $\sqrt{s}=7 \mathrm{TeV}$, J. High Energy Phys. 12 (2013) 039.

[3] G. Aad et al. (ATLAS Collaboration), Measurement of differential production cross-sections for a $Z$ boson in association with $b$-jets in $7 \mathrm{TeV}$ proton-proton collisions with the ATLAS detector, J. High Energy Phys. 10 (2014) 141.

[4] V. Khachatryan et al. (CMS Collaboration), Measurements of the associated production of a $Z$ boson and $b$ jets in $p p$ collisions at $\sqrt{(s)}=8 \mathrm{TeV}$, Eur. Phys. J. C 77, 751 (2017).

[5] M. A. G. Aivazis, J. C. Collins, F. I. Olness, and W.-K. Tung, Leptoproduction of heavy quarks. II. A unified QCD formulation of charged and neutral current processes from fixed-target to collider energies, Phys. Rev. D 50, 3102 (1994).

[6] M. A. G. Aivazis, F. I. Olness, and W.-K. Tung, Leptoproduction of heavy quarks. I. General formalism and kinematics of charged current and neutral current production processes, Phys. Rev. D 50, 3085 (1994).

[7] R. Thorne and R. Roberts, An ordered analysis of heavy flavor production in deep inelastic scattering, Phys. Rev. D 57, 6871 (1998).
[8] R. Thorne and R. Roberts, A practical procedure for evolving heavy flavor structure functions, Phys. Lett. B 421, 303 (1998).

[9] M. Cacciari, M. Greco, and P. Nason, The $P(T)$ spectrum in heavy flavor hadroproduction, J. High Energy Phys. 05 (1998) 007.

[10] S. Forte, E. Laenen, P. Nason, and J. Rojo, Heavy quarks in deep-inelastic scattering, Nucl. Phys. B834, 116 (2010).

[11] S. Forte, D. Napoletano, and M. Ubiali, Higgs production in bottom-quark fusion in a matched scheme, Phys. Lett. B 751, 331 (2015).

[12] M. Bonvini, A. S. Papanastasiou, and F. J. Tackmann, Resummation and matching of $b$-quark mass effects in $b \bar{b} H$ production, J. High Energy Phys. 11 (2015) 196.

[13] S. Forte, D. Napoletano, and M. Ubiali, Higgs production in bottom-quark fusion: Matching beyond leading order, Phys. Lett. B 763, 190 (2016).

[14] M. Bonvini, A. S. Papanastasiou, and F. J. Tackmann, Matched predictions for the $b \bar{b} H$ cross section at the 13 TeV LHC, J. High Energy Phys. 10 (2016) 053.

[15] S. Catani and M. H. Seymour, A general algorithm for calculating jet cross sections in NLO QCD, Nucl. Phys. B485, 291 (1997); Erratum, Nucl. Phys., B510, 503(E) (1998).

[16] S. Dittmaier, A general approach to photon radiation off fermions, Nucl. Phys. B565, 69 (2000).

[17] S. Catani, S. Dittmaier, M. H. Seymour, and Z. Trocsanyi, The dipole formalism for next-to-leading order QCD 
calculations with massive partons, Nucl. Phys. B627, 189 (2002).

[18] P. Kotko and W. Slominski, General mass scheme for jet production in DIS, Phys. Rev. D 86, 094008 (2012).

[19] S. Catani, M. Ciafaloni, and G. Marchesini, Non-cancelling infrared divergences in QCD coherent state, Nucl. Phys. B264, 588 (1986).

[20] S. Catani, Violation of the Bloch-Nordsieck mechanism in general nonabelian theories and SUSY QCD, Z. Phys. C 37, 357 (1988).

[21] R. Doria, J. Frenkel, and J. C. Taylor, Counter example to nonabelian Bloch-Nordsieck theorem, Nucl. Phys. B168, 93 (1980).

[22] C. Di'Lieto, S. Gendron, I. G. Halliday, and C. T. Sachrajda, A counter example to the Bloch-Nordsieck theorem in nonabelian gauge theories, Nucl. Phys. B183, 223 (1981).

[23] Z. Nagy and D. E. Soper, Parton showers with quantum interference, J. High Energy Phys. 09 (2007) 114.

[24] Z. Nagy and D. E. Soper, Parton distribution functions in the context of parton showers, J. High Energy Phys. 06 (2014) 179.

[25] Z. Nagy and D. E. Soper, A parton shower based on factorization of the quantum density matrix, J. High Energy Phys. 06 (2014) 097.

[26] S. Höche and S. Prestel, Triple collinear emissions in parton showers, Phys. Rev. D 96, 074017 (2017).

[27] S. Höche, F. Krauss, and S. Prestel, Implementing NLO DGLAP evolution in parton showers, J. High Energy Phys. 10 (2017) 093.

[28] G. Altarelli and G. Parisi, Asymptotic freedom in parton language, Nucl. Phys. B126, 298 (1977).

[29] S. Dittmaier, A. Kabelschacht, and T. Kasprzik, Polarized QED splittings of massive fermions and dipole subtraction for non-collinear-safe observables, Nucl. Phys. B800, 146 (2008).

[30] S. Frixione and B. R. Webber, Matching NLO QCD computations and parton shower simulations, J. High Energy Phys. 06 (2002) 029.
[31] T. Gleisberg, S. Höche, F. Krauss, M. Schönherr, S. Schumann, F. Siegert, and J. Winter, Event generation with SHERPA 1.1, J. High Energy Phys. 02 (2009) 007.

[32] S. Höche, F. Krauss, M. Schönherr, and F. Siegert, A critical appraisal of NLO + PS matching methods, J. High Energy Phys. 09 (2012) 049.

[33] T. Gehrmann, S. Höche, F. Krauss, M. Schönherr, and F. Siegert, NLO QCD matrix elements + parton showers in $e^{+} e^{-} \rightarrow$ hadrons, J. High Energy Phys. 01 (2013) 144.

[34] S. Höche, F. Krauss, M. Schönherr, and F. Siegert, QCD matrix elements + parton showers: The NLO case, J. High Energy Phys. 04 (2013) 027.

[35] F. Krauss, R. Kuhn, and G. Soff, AMEGIC++ 1.0: A matrix element generator in $\mathrm{C}++$, J. High Energy Phys. 02 (2002) 044.

[36] T. Gleisberg and F. Krauss, Automating dipole subtraction for QCD NLO calculations, Eur. Phys. J. C 53, 501 (2008).

[37] F. Cascioli, P. Maierhöfer, and S. Pozzorini, Scattering Amplitudes with Open Loops, Phys. Rev. Lett. 108, 111601 (2012).

[38] S. Schumann and F. Krauss, A parton shower algorithm based on Catani-Seymour dipole factorisation, J. High Energy Phys. 03 (2008) 038.

[39] M. Cacciari, G. P. Salam, and G. Soyez, The anti- $k(t)$ jet clustering algorithm, J. High Energy Phys. 04 (2008) 063.

[40] M. Cacciari, G. P. Salam, and G. Soyez, FastJet user manual, Eur. Phys. J. C 72, 1896 (2012).

[41] B. M. Waugh et al., HZTool and Rivet: Toolkit and framework for the comparison of simulated final states and data at colliders, arXiv:hep-ph/0605034.

[42] A. Buckley, J. Butterworth, L. Lönnblad, D. Grellscheid, H. Hoeth, J. Monk, H. Schulz, and F. Siegert, Rivet user manual, Comput. Phys. Commun. 184, 2803 (2013).

[43] R. D. Ball et al. (NNPDF Collaboration), Parton distributions for the LHC Run II, J. High Energy Phys. 04 (2015) 040.

[44] S. Dulat, T. J. Hou, J. Gao, M. Guzzi, J. Huston, P. Nadolsky, J. Pumplin, C. Schmidt, D. Stump, and C.-P. Yuan, The CT14 global analysis of quantum chromodynamics, Phys. Rev. D 93, 033006 (2016). 